OPEN ACCESS

Edited by:

George Tsiamis,

University of Patras, Greece

Reviewed by:

Hyung-Kyoon Choi,

Chung-Ang University, South Korea

Yan-Qun Liu,

Shenyang Agricultural University,

China

*Correspondence:

Xiao Zou

xzou@gzu.edu.cn

tThese authors have contributed equally to this work and share first

authorship

Specialty section:

This article was submitted to

Microbial Symbioses,

a section of the journal

Frontiers in Microbiology

Received: 19 February 2021

Accepted: 08 April 2021

Published: 11 May 2021

Citation:

Zeng Z, Mou D, Luo L, Zhong W,

Duan L and Zou X (2021) Different

Cultivation Environments Affect

the Yield, Bacterial Community

and Metabolites of Cordyceps

cicadae. Front. Microbiol. 12:669785.

doi: 10.3389/fmicb.2021.669785

\section{Different Cultivation Environments Affect the Yield, Bacterial Community and Metabolites of Cordyceps cicadae}

\author{
Zhaoying Zeng ${ }^{1,2,3+}$, Dan Mou't1, Li Luo', Wenlin Zhong ${ }^{1}$, Lin Duan' ${ }^{1}$ and Xiao Zou ${ }^{1,2 *}$ \\ ${ }^{1}$ Institute of Fungal Resources, College of Life Sciences, Guizhou University, Guiyang, China, ${ }^{2}$ Key Laboratory of Plant \\ Resource Conservation and Germplasm Innovation in Mountainous Region (Ministry of Education), Guizhou Key Lab \\ of Agro-Bioengineering, College of Life Sciences/Institute of Agro-Bioengineering, Guizhou University, Guiyang, China, \\ ${ }^{3}$ School of Ecology and Environmental Science, Yunnan University, Kunming, China
}

Cordyceps cicadae is an entomogenous fungus with important uses in traditional Chinese medicine. However, its wild resources have not met consumers' demand due to excessive harvesting practices. Artificial cultivation is therefore an important alternative, but research on cultivating $C$. cicadae in natural habitats has not been reported. In this study, we aimed to explore the viability of cultivating C. cicadae in a natural habitat, in the soil of Pinus massoniana forest. We assessed and compared the yield, metabolite contents and bacterial community composition of $C$. cicadae grown in the Antheraea pernyi pupae at different growth stages, and under different cultivation conditions, in the soil of a natural habitat and in sterile glass bottles. Our results showed that cultivating C. cicadae in a natural habitat is feasible, with up to $95 \%$ of pupae producing C. cicadae fruiting bodies. The content of nitrogen compounds (amino acids) in C. cicadae cultivated in a natural habitat was significantly higher than in glass bottles, while the yield and carbon compound (mannitol and polysaccharide) and nucleoside (cordycepin and adenosine) contents were lower. Different bacterial genera were enriched in C. cicadae at different growth stages and cultivation environments, and these bacterial genera were closely related to metabolites contents during growth. This study demonstrated the viability of a novel cultivation method of C. cicadae, which could be used as an alternative to wild stocks of this fungus. These findings provided new insights into the growth mechanism of $C$. cicadae and its interaction with soil microorganisms.

Keywords: Cordyceps cicadae, cultivation in natural habitat, artificial cultivation, metabolite, bacterial community composition, PICRUSt

\section{INTRODUCTION}

Cordyceps cicadae is an entomogenous fungus belonging to the family Claviciptaceae that has been used as a traditional Chinese medicine for over 1600 years (Liu, 2012). It has several host cicadas, such as Cicada flammata Distant, Platypleura kaempferi Fabricius, Cryptotympana pustulata Fabricius, Platylomia pieli Kato, and Oncotympana maculatieollis Motsch (Zeng et al., 2014, 2017), 
and can form fruiting bodies on the surface of such cicada nymphs after they have been parasitized and killed by Isaria cicadae, the anamorphic form of this fungus (Cheng et al., 2006). C. cicadae mainly grows in the soil of bamboo, broad-leaved and evergreen broad-leaved forests, but also grows in mixed coniferous and broad-leaved forests. It is geographically widely distributed in provinces south of the Qinling-Huaihe River in China, including Guizhou, Yunnan, Sichuan, and Zhejiang, as well as in Japan, South Korea, Australia, Brazil, and other countries (Liu, 2012; Chen Z.A. et al., 2014; Zeng et al., 2015).

In China, C. cicadae is used to treat chronic kidney diseases, palpitations and children with seizures, and is also used as a food and tonic (Hsu et al., 2015; Li et al., 2019). Recent studies have found that $C$. cicadae contains metabolites with important pharmacological functions. Examples of these include the carbon compound mannitol, which is a diuretic, exhibits antifree-radical activity, and improves cerebral microcirculation and blood flow (Lin et al., 2016; Zhang et al., 2019); polysaccharides, which are also essential carbon compounds, have antioxidant properties and play a role in treating diabetes and improving immune regulation (Lu et al., 2016; Zhang et al., 2018; Wang et al., 2019); adenosine, a nucleoside with anti-inflammatory, neuroprotective and anti-convulsive properties, which has been shown to improve cell viability and prevent and treat neurodegenerative diseases (Latini and Pedata, 2001; Nakav et al., 2008; Olatunji et al., 2016b); and cordycepin, another nucleoside with anti-cancer, immune regulation, and antioxidant functions (Olatunji et al., 2016a; Zhang et al., 2019). Owing to its use as a medicine and tonic, wild $C$. cicadae has been harvested at unsustainable levels, resulting in a significant population reduction in its natural habitats (Liu et al., 2008). The demand for C. cicadae cannot be met using these practices, and artificial cultivation is necessary to be an alternative to wild C. cicadae and ensure an adequate supply (Hsu et al., 2015; He W.W. et al., 2019; Sun et al., 2019; Nxumalo et al., 2020).

Artificial cultivation techniques for $C$. cicadae include liquid fermentation (Cheng et al., 2006, 2012) and cultivation on cereal medium (Feng, 2002; Zhang et al., 2012; Ke and Lee, 2016) or pupae (Hu et al., 2009; He Y.Q. et al., 2019). Hyphae can only be produced by liquid fermentation, and fruiting bodies only by cereal medium. Cultivation on pupae has become more popular in recent years, because $C$. cicadae with insects and fruiting bodies can be harvested by this way (He W.W. et al., 2019). However, the cultivation of C. cicadae on pupae has mainly been conducted in sterile environments, such as sterilized glass bottles, sand, or soil (Hu et al., 2009; Liu et al., 2018); little research has been conducted on cultivating C. cicadae in natural habitats, despite a preference among consumers for more "natural" production methods (Huang, 2008).

Wild C. cicadae grows in complex natural habitat that are rich in vegetation including Camellia japonica, Camellia oleifera, Pyrus pashia, and Myrica nana (Liu, 2012; Zeng et al., 2015). Researches have shown that the habitat soil, sclerotia, and external mycelial cortices of $C$. cicadae enrich abundant microbial communities and share some of the same bacterial genus, and some ectomycorrhizal fungi have also been detected in sclerotia (Zeng et al., 2019; Mou et al., 2021).
These results suggested that wild C. cicadae populations are ecologically linked to the surrounding plants and soil through microorganisms, which may impact $C$. cicadae growth and production of metabolites. Previous research found that the composition of microbial communities in host varies between different growth environments (Mueller et al., 2020), and changes in microbial community composition in Cordyceps spp. can affect growth and metabolite production of host (Zhang et al., 2010; Qu et al., 2019a,b). Cultivating C. cicadae in natural habitats, rather than under sterile conditions, may therefore be a helpful experimental approach to exploring soil microbial interactions and their potential impact on growth and metabolite production in $C$. cicadae. In addition, we previously demonstrated that the relative abundance of fungi other than Isaria in the sclerotia of C. cicadae was less than $1 \%$, while the bacterial community was more abundant and stable (Zeng et al., 2019; Mou et al., 2021). Given their relative abundance compared with fungi, exploring the composition of bacterial communities in the sclerotia of C. cicadae is a specific priority in this study.

This study therefore aimed to explore the feasibility of cultivating C. cicadae in natural habitats, the bacterial community composition of $C$. cicadae in different cultivation environments, and the relationship between bacterial communities in sclerotia of $C$. cicadae and metabolite production in this medicinally important fungal species. To achieve this, we compared the yield, bacterial community composition and metabolites content of C. cicadae cultivated in aseptic glass bottles versus in the soil of a natural Pinus massoniana forest habitat.

\section{MATERIALS AND METHODS}

\section{Cultivation of Cordyceps cicadae in Two Cultivation Environments}

The Isaria cicadae strain GZUIFR_DJS1 (preserved in Institute of Fungal Resources of Guizhou University) was activated and purified on potato dextrose agar (PDA, $200 \mathrm{~g}$ potato extract, $20 \mathrm{~g}$ glucose, $20 \mathrm{~g}$ agar, 1,000 $\mathrm{mL}$ distilled water) solid growth medium. Purified colonies were inoculated in the center of the PDA medium and cultured upside down at $25^{\circ} \mathrm{C}$ for $5-7$ days, until they produced spores. Spore suspensions [concentration $(C)=5 \times 10^{7}$ spores $/ \mathrm{mL}$ ] were prepared with sterile ultrapure water containing $0.05 \%$ Tween 80 viscous liquid.

Pupae of Antheraea pernyi Guérin-Méneville, sourced from the Yuxi City Artificial Sericulture Base (Liaoning, China), were selected as host insects (cultivation medium) for C. cicadae. Pupae were soaked and scrubbed with 75\% alcohol for $10 \mathrm{~s}$ for body surface sterilization; pupae were rinsed three times with sterile water to remove any residual alcohol, and then dried with sterile tissue. An inoculation of $0.02 \mathrm{~mL}$ spore suspension was made into each pupa at the intersection of the wing and the third somite (Zhang et al., 2016). Inoculated pupae were cultivated in sterile glass bottles at $25^{\circ} \mathrm{C}$, each bottle contained a pupa and added a sterile moist cotton ball for maintaining humidity. After a week, the pupae were completely infected by $I$. cicadae and had become mummified. Each pupa was given one of two possible treatments for 2 weeks (Figure 1): 


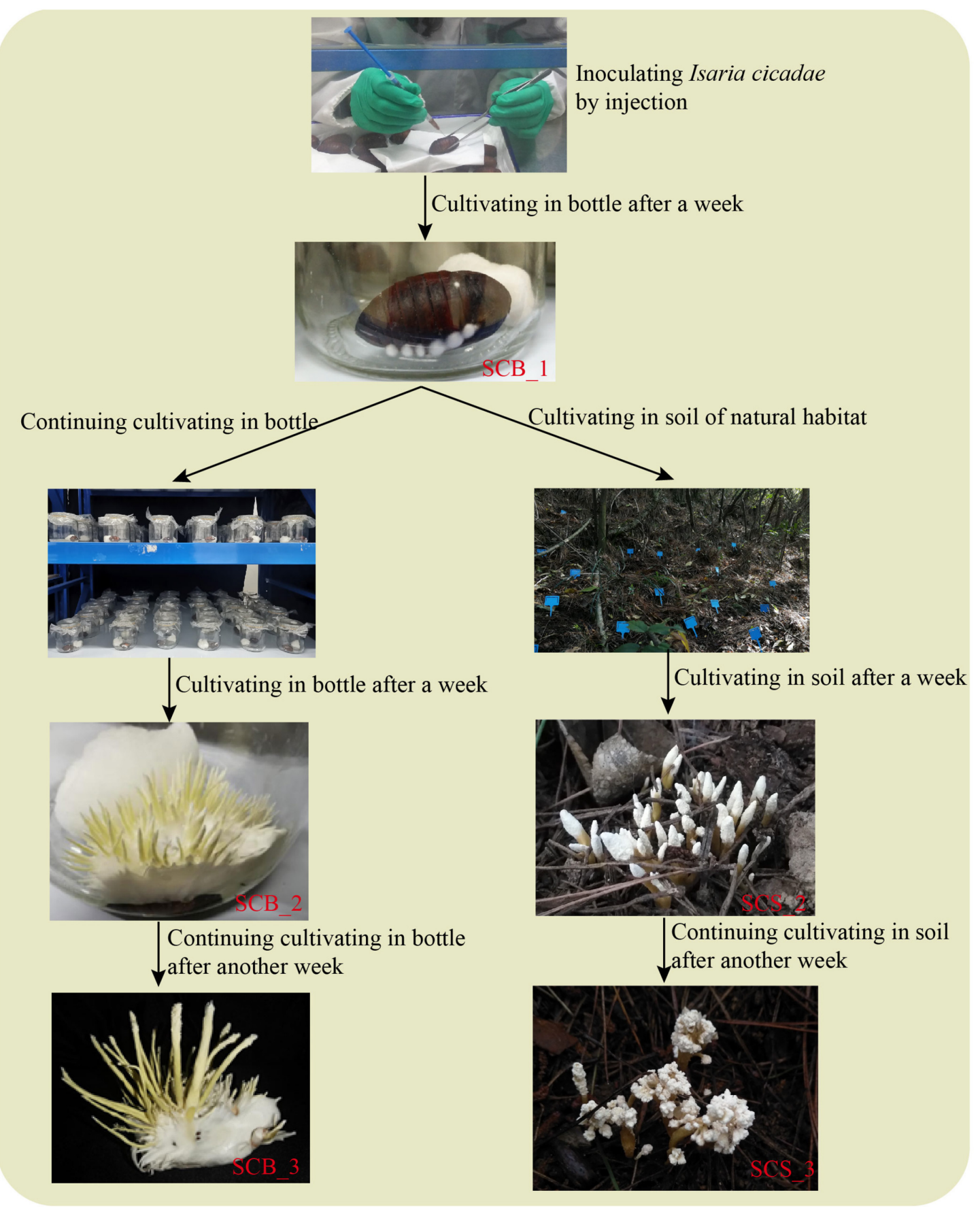

FIGURE 1 | A comparison of Cordyceps cicadae cultivation in sterile glass bottles and in the soil of natural habitats.

either their cultivation in glass bottles was continued (hereafter referred to as $\mathrm{CB}$ ), or they were cultivated in the soil of natural habitat under a Pinus massoniana forest (CS). The experimental cultivation site for natural habitat used in this study was located in the West Campus of Guizhou University (Guizhou, China) and measured approximately $100 \mathrm{~m}^{2}$; a detailed description of the habitat is in Supplementary Table 1. Mummified pupae were cultivated in natural habitat under a layer of covering soil approximately $1.5 \mathrm{~cm}$ thick, and an interval between any two pupae of $20 \mathrm{~cm}$ or more.

\section{Experiment 1: Effects of Two Cultivation Environments on the Yield of C. cicadae}

In April 2019, 150 pupae were inoculated and cultivated in sterile glass bottles for 1 week. After removing non-mummified 
pupae, 120 mummified pupae were randomly divided into six groups of 20; three groups in CB and three in CS. Under both treatment conditions for 2 weeks, $C$. cicadae fruiting bodies did not grow anymore and began to produce spores, at this point, $C$. cicadae were considered mature; then we harvested all C. cicadae and washed any soil from CS pupae using tap water. Then, the proportion of pupae producing C. cicadae fruiting bodies (i.e., the number of pupae producing fruiting bodies divided by the total number) was calculated. The average proportion of pupae with fruiting bodies across all three groups was then calculated for CB and CS separately. After removing any non-mummified pupae and any C. cicadae damaged in the process of washing soil from CS pupae, the fresh weight, fruiting body number, length and diameter of 53 out of 55 C. cicadae in CB and 39 out of 57 in CS were measured. Finally, we compared the relative yield between in CB and CS by 100 cicadas i.e., $100 \times$ the proportion of pupae producing $C$. cicadae fruiting bodies $\times$ mean fresh weight per C. cicadae).

\section{Experiment 2: Effects of Two Cultivation Environments on the Bacterial Community and Metabolites of C. cicadae}

In September 2019, 250 pupae were inoculated and cultivated in sterile glass bottles for 1 week. After removing non-mummified pupae, 200 mummified pupae were randomly divided into two groups and cultivated for 2 weeks under CB and CS treatment conditions, respectively. Since the pupae were inoculated with spore suspension, the sclerotia of nine randomly selected pupae were collected for bacterial community analysis each week, with three pupae combined in each of three replicates. Only pupae in the $\mathrm{CB}$ treatment condition were taken in week 1 ; in subsequent weeks, pupae in both CB and CS treatment conditions were taken. Samples were coded according to the week they were taken, e.g., samples from the second week were SCB_2 and SCS_2. Any soil on the CS pupae was washed off with tap water before the external mycelial layer and fruiting body were stripped off, leaving only the mummified pupae. These pupae were soaked in $75 \%$ alcohol for $2 \mathrm{~min}$, then soaked in $2.5 \%$ sodium hypochlorite solution for $20 \mathrm{~s}$, washed with sterile water five times, and dried with sterile tissue (Xia et al., 2015). Each pupa was crosscut in half on a clean laboratory bench, and the C. cicadae sclerotia were picked out with an inoculation needle and placed in sterile centrifuge tubes. Each sample was flash-frozen with liquid nitrogen, ground, and stored at $-80^{\circ} \mathrm{C}$ until the DNA could be extracted. In the third week, the remaining $C$. cicadae in the two treatments were harvested, washed to remove soil, and then dried by dry oven at $80^{\circ} \mathrm{C}$. Fruiting bodies were collected, ground and sifted through a 100 mesh screen, and used to detect the contents of five metabolites in C. cicadae.

\section{Detection of Metabolites}

In this study, we measured the content of adenosine, cordycepin, polysaccharides, and mannitol in C. cicadae and the content and type of amino acids in C. cicadae fruiting bodies. Amino acids were determined using the Hitachi L-8800 high speed amino acid analyzer, according to the national standard protocol GB 5009.124-2016. Mannitol content was determined by colorimetry according to a previously established method (San et al., 2010): 50\% ethanol was used to extract mannitol using ultrasonic method, then sodium periodate solution, L-rhamnose solution and Nash reagent were used to perform the coloration reaction; absorption values were then detected at $412 \mathrm{~nm}$ using an ultraviolet spectrophotometer. Polysaccharide content was determined using a sulfuric acidphenol method according to the national agricultural standard NY/T 1676-2008. Polysaccharides were precipitated in ethanol, and the resulting furfural derivatives of the polysaccharides were dehydrated in concentrated sulfuric acid and condensed with phenol, forming orange-red compounds with a color intensity proportional to the concentration of polysaccharides in the solution. Colorimetric quantification was carried out at $490 \mathrm{~nm}$ by an ultraviolet spectrophotometer. Cordycepin and adenosine were determined using high performance liquid chromatography according to national agricultural standard NY/T 2116-2012. The chromatographic column used in this study was the Shim-pack VP-ODS 5 micron $250 \mathrm{~mm} \times 4.6 \mathrm{~mm}$. The mobile phase was water and acetonitrile with 95:5 volume (v/v) ratio at a mobile speed of $1.0 \mathrm{~mL} / \mathrm{min}$. The temperature of chromatographic column was maintained at $35^{\circ} \mathrm{C}$. The contents of cordycepin and adenosine were measured using a UV detector at $260 \mathrm{~nm}$. We conducted three replicates for the measurement of each metabolite.

\section{Bacterial DNA Extraction, PCR Amplification, and Sequencing}

Bacterial DNA was extracted using the E.Z.N.A. ${ }^{\circledR}$ Soil DNA Kit (Omega Bio-tek), and the concentration and purity of DNA were assessed by electrophoresis on $1.0 \%$ weight/volume agarose gels. The primers used to amplify the V4 variable region of the bacterial 16s RNA gene were: 515F (5'-GTGCCAGCMGCCGCGGTAA-3') and 806R (5'GGACTACHVGGGTWTCTAAT-3') (Zhou et al., 2020). DNA amplification was carried out using the ABI GeneAmp 9700 polymerase chain reaction (PCR) instrument. The PCR parameters were as follows: initial denaturation for $3 \mathrm{~min}$ at $95^{\circ} \mathrm{C}$; denaturation, annealing and extension at $30 \mathrm{~s}$ at $95^{\circ} \mathrm{C}$, $30 \mathrm{~s}$ at $55^{\circ} \mathrm{C}$, and $45 \mathrm{~s}$ at $72^{\circ} \mathrm{C}$ for 30 cycles; then final extension for $10 \mathrm{~min}$ at $72^{\circ} \mathrm{C}$, then $10^{\circ} \mathrm{C}$ until the reaction stopped. The amplification products were sent to Shanghai Majorbio Biopharm Technology Co., Ltd. (Shanghai, China) for sequencing on the Illumina MiSeq sequencing platform.

\section{Bioinformatics and Data Analysis}

Fast Length Adjustment of SHort reads (FLASH) and Quantitative Insights Into Microbial Ecology (QIIME) software programs were used to merge and quality-filter raw reads from the original DNA fragments (Caporaso et al., 2010; Magoc and Salzberg, 2011). Effective reads were obtained by using a UCHIME algorithm to identify and remove chimeric sequences. Quality sequences were then clustered into operational taxonomic units (OTUs) with a $>97 \%$ similarity cutoff value using the UPARSE algorithm (Edgar et al., 2011). 

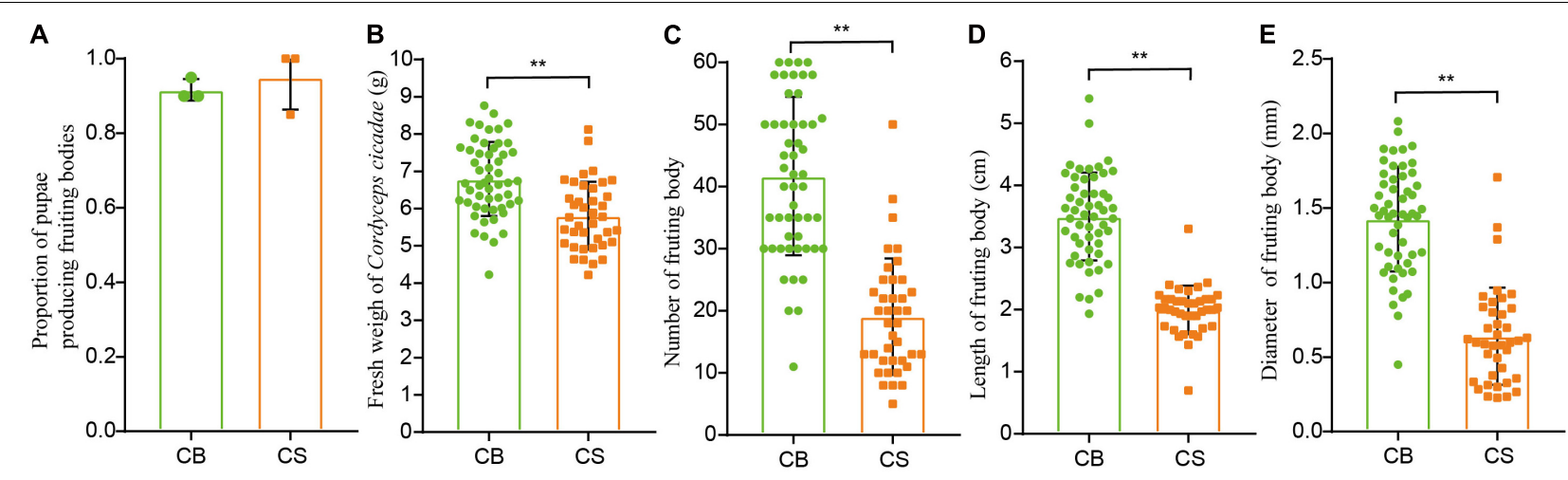

FIGURE 2 | The effects of two cultivation environments on yield measurements in Cordyceps cicadae. (A) proportion of pupae producing fruiting bodies; (B) fresh weight of Cordyceps cicadae; (C) number of fruiting bodies; (D) length of fruiting bodies; (E) diameter of fruiting bodies. CB, cultivation in glass bottles; CS, cultivation in the soil of a natural habitat. ${ }^{* \star} p<0.01$, according to non-parametric Mann-Whitney $U$-tests, error bars depict the standard deviation.

The Basic Logical Alignment Search Tool (BLAST) was then used to compare representative sequences for each OTU with sequences in the SILVA database to obtain the taxon of each OTU (Quast et al., 2013).

$\alpha$-diversity of bacterial communities in each sample was represented by Shannon, Chaol and Coverage indices, calculated after reads were normalized to the minimum reads (30, 665 reads) in each sample. Phylogenetic Investigation of Communities by Reconstruction of Unobserved States (PICRUSt) ${ }^{1}$ software was used to predict the functional profile of bacterial communities using 16S ribosomal ribonucleic acid (rRNA) marker gene sequences (Langille et al., 2013). We then calculated the abundance of Kyoto Encyclopedia of Genes and Genomes (KEGG) pathways and functions at the OTU level in each sample.

The Kolmogorov-Smirnov tests were used to verify the normality of data. Student's $t$-tests and non-parametric MannWhitney $U$-tests were used to identify differences in yield and abundance of KEGG pathways and functions between $\mathrm{CB}$ and CS treatment conditions that the data were normal or not, respectively. One-way analysis of variance (ANOVA) and post hoc comparisons were performed using Duncan's Multiple Range test to compare $\alpha$-diversity among the different groups. The Spearman's rank correlation coefficient between contents of metabolites mentioned above and the top 30 most abundant bacterial genera was calculated and then visualized by the "pheatmap" package in the R software program. Visualizations of the yield index, the $\alpha$-diversity index and the bacterial community composition were produced using GraphPad Prism 7 (GraphPad Software Inc., San Diego, CA, United States).

\section{RESULTS}

\section{Lower Yield of C. cicadae Under Natural Habitat Compared With Sterile Bottle}

The proportion of pupae producing fruiting bodies was similar between CS and CB cultivation environments (92 $\pm 14 \%$ in

${ }^{1}$ http://picrust.github.io/picrust/
CS and $95 \pm 9 \%$ in $\mathrm{CB}$, Figure $2 \mathrm{~A}$ ), indicating that $C$. cicadae can be cultivated in the soil of a natural forest habitat as in glass bottle. However, the fresh weight, number, length and diameter of fruiting bodies in CS were all significantly lower than those in $\mathrm{CB}(p<0.01$, Figures $2 \mathrm{~B}-\mathrm{E})$, and the relative yield in CS (534.52g/100 cicada) was also lower than that in CB (645.05g/100 cicada, Supplementary Table 2), indicating that the overall growth of C. cicadae in CB was greater than in CS.

\section{Contents of Metabolites in C. cicadae Differed Between Two Cultivation Environments}

In total, 17 amino acids were detected in C. cicadae cultivated in both environments (Figure 3A). The total amino acid content of C. cicadae in CS $(28.87 \pm 0.057 \%)$ was significantly higher than in CB $(26.01 \pm 0.029 \%, p<0.01$, Figure $3 B)$. The contents of aspartic acid, threonine, serine, glutamic acid, glycine, alanine, cysteine, valine, methionine, isoleucine, phenylalanine, histidine, lysine, arginine, and leucine of $C$. cicadae were significantly higher in CS than in CB $(p<0.01)$. Conversely, the proline content in CS $(2.51 \pm 0.005 \%)$ was significantly lower than that in $\mathrm{CB}(3.04 \pm 0.015 \%, p<0.01)$. Cordycepin and polysaccharide contents of $C$. cicadae were slightly lower in CS than in CB. Adenosine and mannitol contents were significantly lower in CS than CB $(p<0.01$, Figure 3B). These results indicate that cultivating $C$. cicadae in natural habitats increases the production of nitrogen compounds (amino acids), but decreases that of carbon compounds (polysaccharides and mannitol), and nucleosides (cordycepin and adenosine).

\section{Bacterial Communities in Sclerotia of C. cicadae Were Higher Richness When Cultivated in Natural Habitat Compared With Sterile Bottle}

A total of 2,931,602 raw sequences were detected. After filtering, $1,465,801$ effective reads were obtained, with $30,665-92,371$ in each sample. The coverage index of samples $(>0.99$ for all 


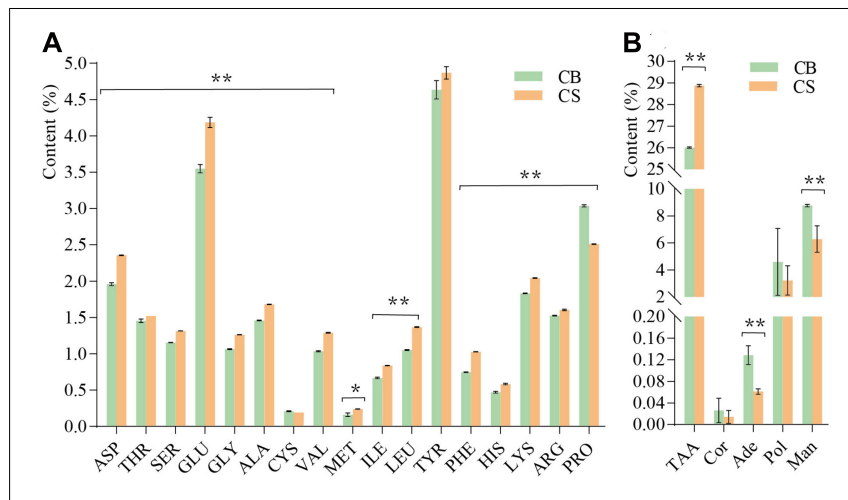

FIGURE 3 | The effects of two cultivation environments on metabolites produced by Cordyceps cicadae. (A) content of 17 amino acids; (B) content of five metabolites. ASP, aspartic acid; THR, threonine; SER, serine; GLU, glutamic acid; GLY, glycine; ALA, alanine; CYS, cysteine; VAL, valine; MET, methionine; ILE, isoleucine; LEU, leucine; TYR, tyrosine; PHE, phenylalanine; HIS, histidine; LYS, lysine; ARG, arginine; PRO, proline; TAA, total amino acid; Cor, cordycepin; Ade, adenosine; Pol, polysaccharide; Man, mannitol. Error bars depict the standard deviation $\left({ }^{*} p<0.05,{ }^{* *} p<0.01\right.$, according to Student's $t$-tests)
Achromobacter (50.24 $\pm 46.77 \%)$ and Serratia (32.34 $\pm 56.01 \%)$ were dominant in SCB_3. In C. cicadae cultivated in natural habitats, Pseudomonas $(61.80 \pm 18.32 \%)$ and Achromobacter $(80.75 \pm 15.18 \%)$ were the dominant genera in SCS_2 and SCS_3, respectively. The relative abundance of Achromobacter gradually increased from $0.02 \pm 0.012 \%$ to $80.75 \pm 15.18 \%$ with the growth of C. cicadae, which showed significantly different relative abundance among groups $(p<0.05)$. The relative abundance of Pseudomonas, Enterococcus, Ralstonia, Rhodococcus, and Amaricoccus were also significantly different among groups. Pseudomonas, Ralstonia, Rhodococcus, and Amaricoccus had a significantly higher relative abundance in SCS_2 than at other stages in both environments (Rhodococcus, $p<0.05$; other $p<0.01$ ), while Enterococcus had significantly higher relative abundance in $S C B \_1$ than at other stages in both environments $(p<0.05)$. Cedecea only had higher relative abundance $(33.18 \pm 23.46 \%)$ in SCB_2, but a low relative abundance $(0.001 \pm 0.00095 \%-1.09 \pm 1.55 \%)$ at other stages in both environments (Figure 5B). These data show that the bacterial community composition and dominant genera in the sclerotia of $C$. cicadae vary significantly between cultivation environments and growth stages.

\section{Predictive Functional Profiling of Bacterial Communities}

The functions of bacterial communities were predicted by PICRUSt to determine the changes in relative abundance of bacterial OTUs related to functions in samples from different cultivation environments and time points (Figure 6). Bacteria OTUs related to metabolism had the highest relative abundance (62.80-66.33\%) at the KEGG pathway Level 1, followed by environmental information processing (11.10$14.06 \%)$, genetic information processing (6.04-12.14\%), human diseases (4.59-5.29\%), cellular processes (2.99-4.04\%), and organismal systems (1.71-2.36\%).

At the KEGG pathway Level 2, bacteria OTUs with function related to carbohydrate metabolism (11.98-18.53\%), global and overview maps (10.10-11.81\%), Amino acid metabolism (7.47$12.58 \%)$ in metabolism of Level 1 and membrane transport (6.55-10.11\%) in environmental information processing of Level 1 showed higher relative abundance than other functions. The relative abundance of bacteria OTUs related to glycan biosynthesis and metabolism and carbohydrate metabolism were significantly lower in CS $(1.26 \pm 0.06 \%$ and $12.42 \pm 0.31 \%$, respectively) than in $\mathrm{CB}(1.57 \pm 0.29 \%$ and $14.28 \pm 2.04 \%$, respectively; both $p<0.05)$. Conversely, in CS compared with $\mathrm{CB}$, the relative abundance of bacteria OTUs related to amino acid metabolism (12.08 $\pm 0.39 \%$ versus $10.44 \pm 1.59 \%)$, xenobiotic biodegradation and metabolism ( $4.12 \pm 0.50 \%$ versus $3.04 \pm 1.02 \%)$, metabolism of terpenoids and polyketides (1.67 $\pm 0.03 \%$ versus $1.46 \pm 0.15 \%)$, and biosynthesis of other secondary metabolites $(1.60 \pm 0.04 \%$ versus $1.42 \pm 0.13 \%)$ were all significantly higher (all $p<0.05$ ). These results suggested the relative abundance of bacteria OTUs with functions related to metabolism were significantly different between two cultivation environments, and further indicated the potential 

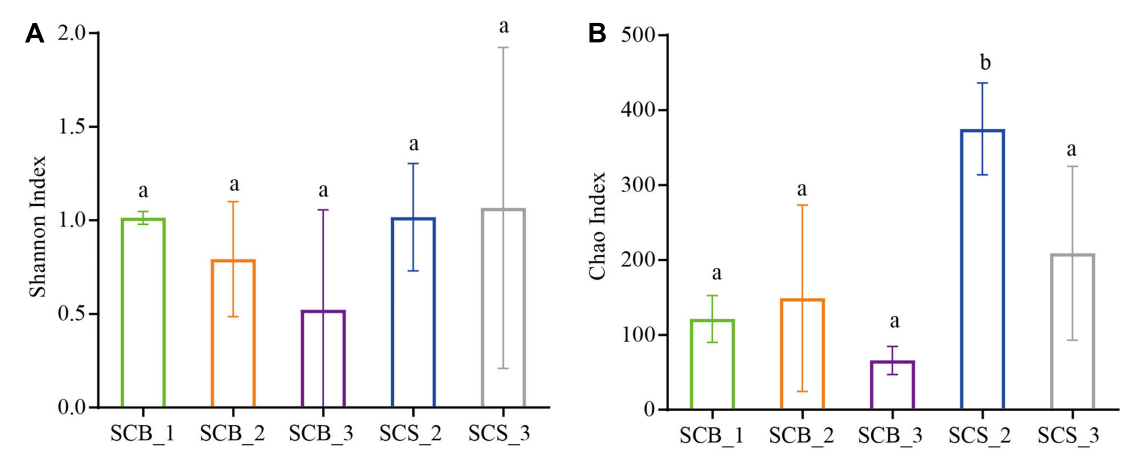

FIGURE 4 | Diversity index of bacterial communities in sclerotia of Cordyceps cicadae under the two cultivation environments (SCB, sclerotia of Cordyceps cicadae cultivating in glass bottles; SCS, sclerotia of Cordyceps cicadae cultivating in the soil of a natural habitat) at 1, 2, and 3 weeks (denoted by _ 1, _2, and _3, respectively) after inoculation. (A) Shannon index of diversity; (B) Chao1 index of richness. Different lowercase letters indicate significant difference ( $0<0.05)$. Error bars depict the standard deviation.

correlation between the changes of metabolite content and bacterial community composition in C. cicadae cultivated in different cultivation environments.

\section{The Relationship Between Bacterial Community and Metabolite}

To further understand the relationship between bacterial communities and metabolites contents in cultivated C. cicadae, we calculated the Spearman's rank correlation coefficient between the top 30 most abundant genera and the contents of 22 metabolites, including 17 amino acids (Figure 7). There was a significant correlation between most bacterial genera and metabolite contents, especially those of amino acids.

Total amino acids, tyrosine, arginine, lysine, phenylalanine, valine, and leucine were all significantly positively correlated with Rhodococcus, Faecalibacterium, Enterobacter, Bacteroides, Candidatus Rhabdochlamydia, Brevundimonas, Amaricoccus, Ralstonia, and Bifidobacterium, but negatively correlated with Cosenzaea (all $p<0.05$ at least). Pseudomonas, BurkholderiaCaballeronia-Paraburkholderia, and Acinetobacter were all significantly correlated with total amino acids, arginine, lysine, phenylalanine, valine and leucine (all $p<0.05$ at least). Proline were significantly positively correlated with Serratia, Bacillus, and Enterococcus, but negatively correlated with Achromobacter and Faecalibacterium (all $p<0.05$ at least). There was a significant positive correlation between polysaccharide and Cosenzaea $(p<0.01)$. Cordycepin was significantly negatively correlated with numerous genera, including Rhodococcus, Faecalibacterium, Enterobacter, Bacteroides, Candidatus_Rhabdochlamydia, Brevundimonas, Amaricoccus, Ralstonia, Acinetobacter, Bifidobacterium, and Lactobacillus, but significantly positively correlated with Lactococcus (all $p<0.05$ at least). Adenosine were significantly positively correlated with Cosenzaea $(p<0.01)$, but negatively correlated with Rhodococcus and Faecalibacterium (both $p<0.05$ ). Mannitol were significantly positively correlated with Cosenzaea and Enterococcus, but significantly negatively correlated with Rhodococcus, Faecalibacterium, Enterobacter, and Brevundimonas (all $p<0.05$ at least).

\section{DISCUSSION}

This study confirmed that it is feasible to cultivate C. cicadae in the soil of a natural forest habitat, but the yield was lower than that of C. cicadae cultivated in sterile glass bottles. Our research also showed that different growth environments appear to affect the growth, bacterial community composition and metabolite content of C. cicadae, and bacterial community composition was correlated with metabolites content of C. cicadae.

Cultivating C. cicadae in natural habitats could help to meet consumer demand for natural products and reduce the exploitation of wild C. cicadae (Huang, 2008). However, in our study, the yield of $C$. cicadae cultivated in natural habitats was markedly reduced. Some research found that changes in growth environments can result in some resources being allocated to build a defense system to facilitate growth (Feng, 2008; Simon et al., 2010); it is therefore possible that, in the soil of their natural habitats, C. cicadae may also use resources to build a defense system to reduce the adverse effects of natural competitors and microorganisms on their growth. This would leave fewer resources allocated to growth of C. cicadae, which could have resulted in the reduced yield we observed in our study.

All organisms lived in complex microbial ecosystems and formed a close relationship with diverse microorganisms; changes in the growth environment can affect the microbial community associated with the host (Mueller et al., 2020). In this study, different growth environments were shown to affect bacterial community composition and dominant bacterial genera in the sclerotia of C. cicadae. Pseudomonas was significantly enriched in sclerotia of C. cicadae cultivated in the soil of a natural habitat. Researches showed that Pseudomonas was abundant in soil (Li et al., 2013), and Mou et al. (2021) found that Pseudomonas was the dominant genus in the sclerotia and external mycelial cortices of wild C. cicadae. We thought therefore the Pseudomonas in C. cicadae cultivated in a natural habitat may originated from the soil; if this is the case, this indicates that $C$. cicadae may communicate with microorganisms in the soil within its natural habitat. Pseudomonas spp., regarded as broad-host-range entomopathogenic bacteria, are known to 

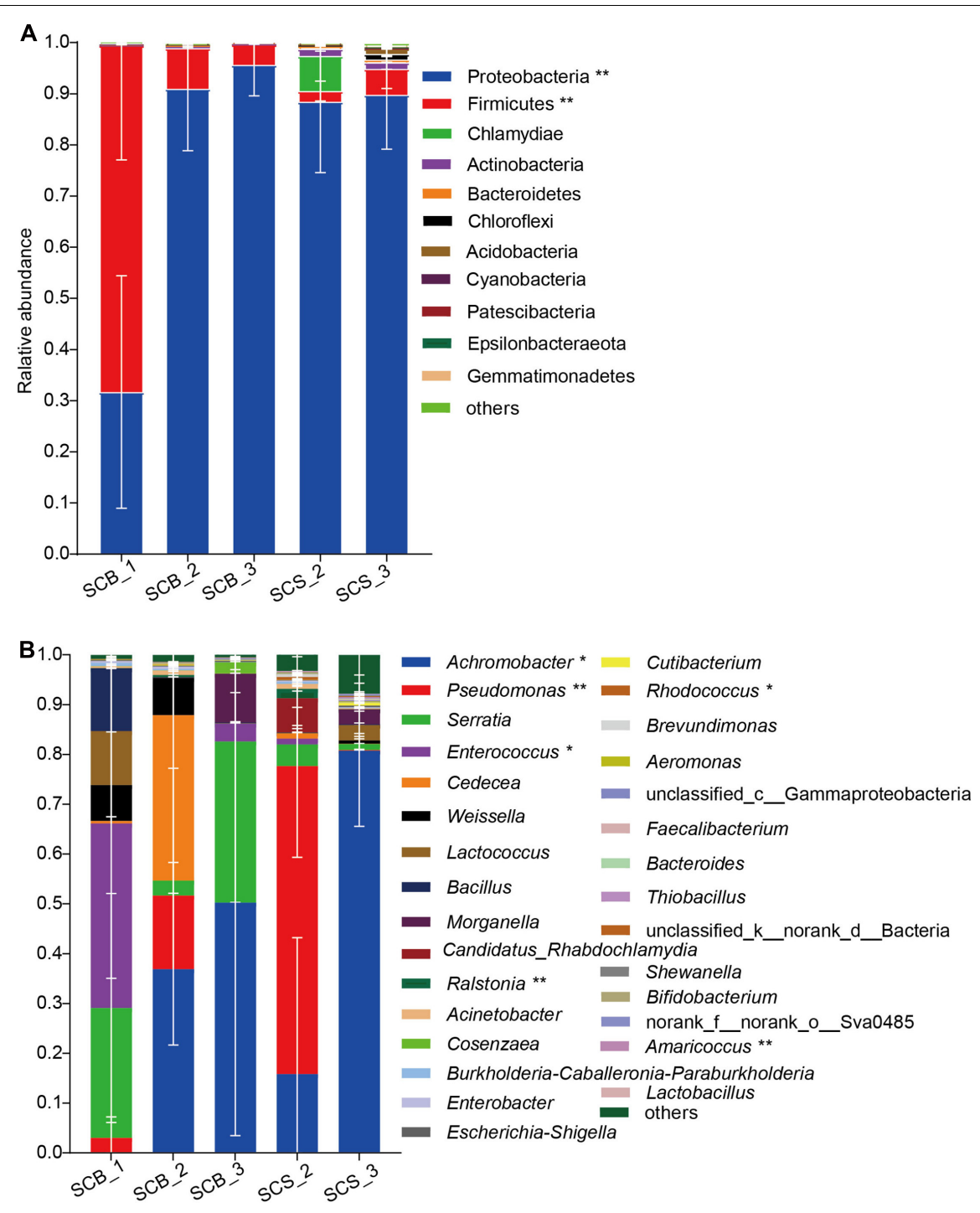

FIGURE 5 | Bacterial community composition in sclerotia of Cordyceps cicadae at different growth stage in both cultivation environments (explanations for abbreviations in $X$ axis are shown in Figure 4). (A) Phyla with relative abundance greater than $0.1 \%$; (B) Top 30 most abundant genera (* and ${ }^{* \star}$ denotes significance between all growth stages in both environments of $p<0.05$ and $p<0.01$, respectively, according to ANOVA, error bars depict the standard deviation).

exhibit insecticidal activity toward certain agricultural pests (Chen W.J. et al., 2014). This could potentially benefit the growth of C. cicadae, for example by promoting the mummification process, as previously observed in Chinese Cordyceps (Wu et al., 2020). In addition, in this study, the sclerotia of C. cicadae cultivated in glass bottles was rich in Cedecea bacteria, but this genus had a very low relative abundance in sclerotia of C. cicadae cultivated in natural habitats. Previous researches isolated and detected Cedecea in C. cicadae $(\mathrm{Qu}$ et al., 2019a,b); however, another study did not detect any Cedecea in sclerotia of C. cicadae (Mou et al., 2021), which indicated the abundance of Cedecea in C. cicadae is unstable and may be affected by the growth environment. Furthermore, in our study, bacterial community composition varied with the time point (and therefore growth stage) of C. cicadae, similar to previously published research (Wu et al., 2020). In particular, the relative abundance of Enterococcus gradually decreased with the growth of C. cicadae, suggesting that I. cicadae injected into the pupae may have inhibited some bacterial genera.

In this study, we found that the changes in relative abundance of bacteria OTUs related to some functions were consistent with changes in metabolite content: for example, the relative abundance of bacteria related to amino acid metabolism in C. cicadae in CS increased significantly than in $\mathrm{CB}$, along with the content of total amino acids in this cultivation environment. The relative abundance of bacteria related to carbohydrate metabolism and glycan biosynthesis and metabolism in CS decreased significantly than in $\mathrm{CB}$, along with the content of carbon compound (mannitol and polysaccharides) in this 


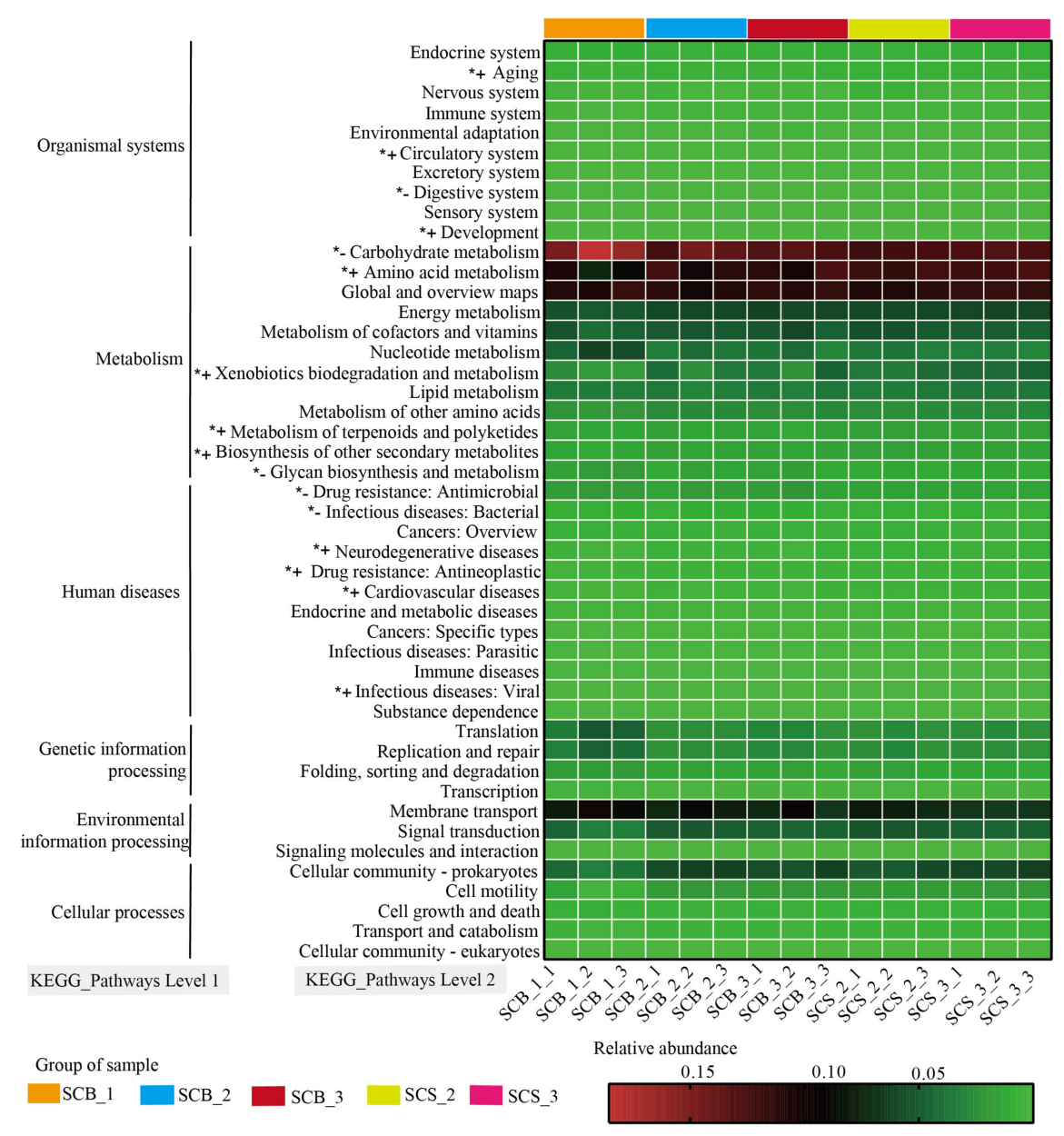

FIGURE 6 | Variation in KEGG metabolic pathways in functional bacterial communities of Cordyceps cicadae cultivated in different cultivation environments (SCB, sclerotia of Cordyceps cicadae cultivating in glass bottles; SCS, sclerotia of Cordyceps cicadae cultivating in the soil of a natural habitat) at 1 , 2, and 3 weeks after inoculation (_1, _2, and _3, respectively). The final number in each sample label represents the replicate number of each sample ( 1 , _2, and _3, respectively). *+ denotes the relative abundance of bacteria related to the function is significantly higher in CS than that in $\mathrm{CB},{ }^{*-}$ represents the relative abundance of bacteria related to the function significantly lower in CS than that in CB $(p<0.05$, according to Student's $t$-test).

cultivation environment. Researches showed culture conditions (Cheng et al., 2012; Lee et al., 2019), nutrient sources (Mao et al., 2005; Chunyu et al., 2019), different strains (Kang et al., 2017), and hosts (He Y. et al., 2019) can affect the level of metabolites, but these factors were consistent between two cultivation environment in this study. As a result, the main reason for different level of metabolite in two cultivation environment may be caused by the difference of microbial community composition and related functions (Figures 5, 6). Studies on food fermentation also showed that metabolite production related closely to bacterial community composition in the progress of fermentation (He and Chung, 2020; Zang et al., 2020). Some studies also reported that endophytic microbes in Cordyceps spp. may affect the production of metabolites (Zhang et al., 2010; Qu et al., 2019a) and microbial co-culture can increase significantly the level of some metabolites (Akone et al., 2016; $\mathrm{Yu}$ et al., 2019). Therefore, further researches on determining which microbial species involve in the production of cordycepin, polysaccharide, adenosine, mannitol or amino acid and then using beneficially synthetic community or fungal-bacterial coculture to improve the level of metabolites in $C$. cicadae are necessary. The amino acid content of edible mushrooms was known to be correlated with freshness and sweetness (Mau et al., 2001), which may explain why consumers prefer edible mushrooms grown in a natural habitat. In addition, the nitrogen compounds content of C. cicadae in natural soil habitats increased, while the carbon compounds decreased, which may suggest that $C$. cicadae may exchange carbon and nitrogen elements in the soil, similar function to Metarhizium robertsii (Behie et al., 2017; Barelli et al., 2019). We therefore speculated C. cicadae may exchange carbon and nitrogen elements in soil via endophytic bacteria to affect the content of carbon and nitrogen compounds in $C$. cicadae, which may potentially result in adding a branch to the carbon and nitrogen cycle in the ecosystem, entomogenous fungi $C$. cicadae is involved in carbon and nitrogen cycling in the soil. 


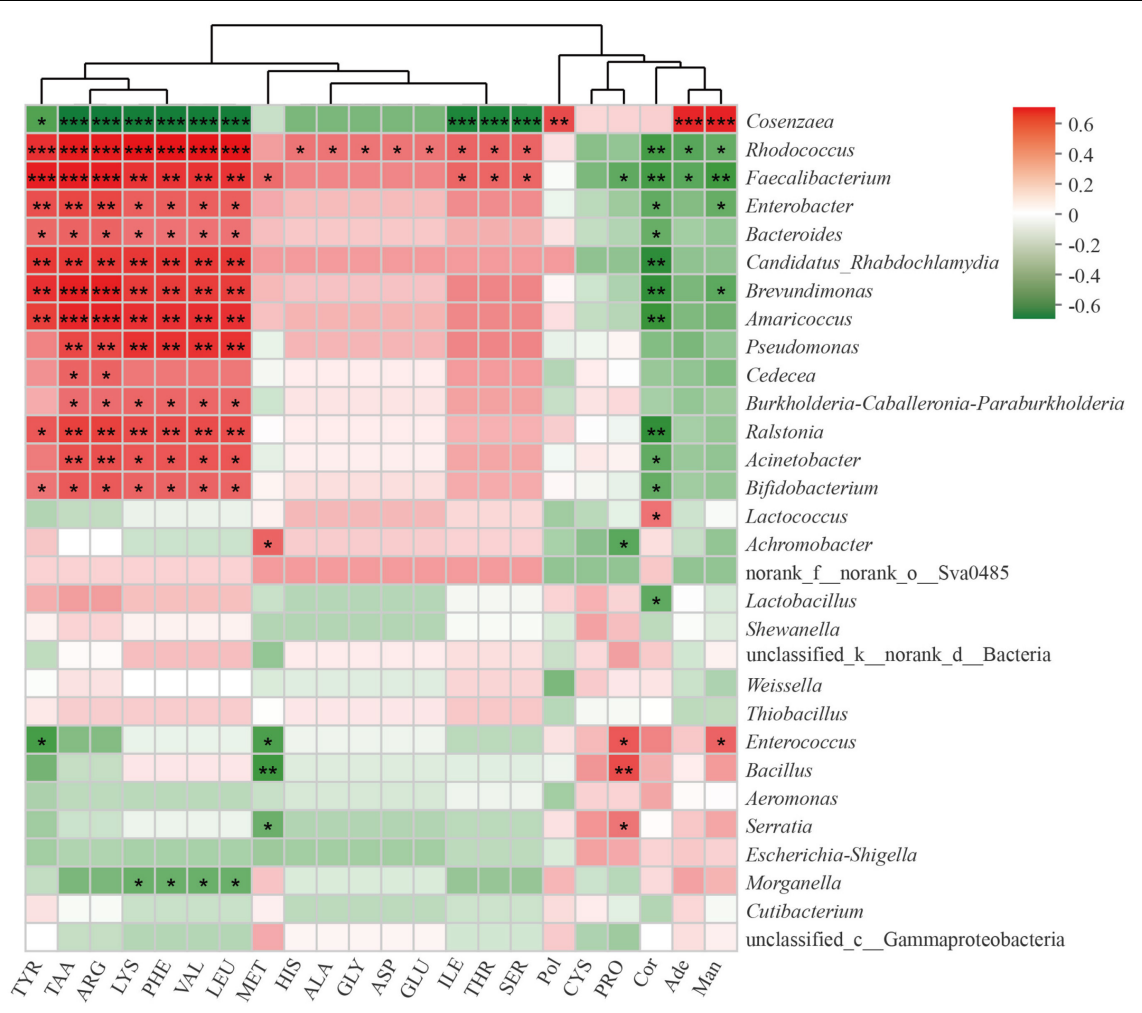

FIGURE 7 | Correlation of top 30 most abundant bacteria genera with metabolites in Cordyceps cicadae. The strength (Spearman's $\rho$ value) of correlations are shown here as different shades of red (positive correlation) and green (negative correlation). Explanations for abbreviations in $X$ axis are shown in Figure $\mathbf{3}$. ${ }^{\star} 0.01<p \leq 0.05 ;{ }^{* \star} 0.001<p \leq 0.01 ;{ }^{* \star *} p \leq 0.001$.

Compared with the contents of metabolites in wild C. cicadae reported in other studies (Ge et al., 2007; Zhang et al., 2017), the total amino acids, polysaccharides and mannitol contents of C. cicadae in our study were higher regardless of how they were cultivated. C. cicadae cultivated in a natural habitat could achieve microbial communication in soil and be favored by consumer, it therefore be an alternative to the wild. Given the apparent close relationship between bacterial community composition and metabolites of $C$. cicadae, we will further isolate, test and screen microorganisms in future studies to identify which species may be beneficial to the growth and metabolite production of cultivated C. cicadae, so that the yield and metabolites contents could be improved by beneficial interaction between microorganisms.

\section{CONCLUSION}

Our study demonstrated for the first time to our knowledge that cultivating C. cicadae in natural habitat soil is feasible, but further studies are required to improve yield. This cultivation method appeared to increase nitrogenous compound (amino acid) and decrease carbonaceous compound (mannitol and polysaccharides) content, possibly through the interaction of C. cicadae with microorganisms in the soil. Metabolite production was found to be closely related to the bacterial community during $C$. cicadae growth; $C$. cicadae may therefore also participate in the carbon and nitrogen cycle in natural ecosystems.

\section{DATA AVAILABILITY STATEMENT}

The datasets presented in this study can be found in online repositories. The names of the repository/repositories and accession number(s) can be found below: https://www.ncbi.nlm. nih.gov/, PRJNA698306.

\section{AUTHOR CONTRIBUTIONS}

ZZ designed and performed the experiments, analyzed the data, and wrote the manuscript. DM designed and performed the experiments and analyzed the data. LL, WZ, and LD collected the samples and performed the experiments. XZ designed the experiments and wrote the manuscript. All authors have read and approved the submission of the manuscript.

\section{FUNDING}

This work was supported by the National Natural Science Foundation of China (31860037), the Youth Science and Technology Talent Project of Guizhou Province ([2018]5781), 
the Science and Technology Plan Projects of Guizhou Province (Qian Ke He Support [2019]2405), and (Qian Ke He Foundation [2020]1Z009).

\section{ACKNOWLEDGMENTS}

We thank Jiaxi Zhou, Chunbo Dong, Yeming Zhou, and Jiaojiao $\mathrm{Qu}$ at Guizhou University for providing advice on data analysis and manuscript preparation. We also thank Guochun

\section{REFERENCES}

Akone, S. H., Mándi, A., Kurtán, T., Hartmann, R., Lin, W. H., and Daletos, G. (2016). Inducing secondary metabolite production by the endophytic fungus Chaetomium sp. through fungal-bacterial co-culture and epigenetic modification. Tetrahedron 72, 6340-6347. doi: 10.1016/j.tet.2016.08.022

Barelli, L., Behie, S. W., and Bidochka, M. J. (2019). Availability of carbon and nitrogen in soil affects Metarhizium robertsii root colonization and transfer of insect-derived nitrogen. FEMS Microbiol. Ecol. 95:fiz144. doi: 10.1093/femsec/ fiz144

Behie, S. W., Moreira, C. C., Sementchoukova, I., Barelli, L., Zelisko, P. M., and Bidochka, M. J. (2017). Carbon translocation from a plant to an insect-pathogenic endophytic fungus. Nat. Commun. 8:14245. doi: 10.1038/ ncomms 14245

Caporaso, J. G., Kuczynski, J., Stombaugh, J., Bittinger, K., Bushman, F. D., Costello, E. K., et al. (2010). QIIME allows analysis of high-throughput community sequencing data. Nat. Methods 7, 335-336. doi: 10.1038/nmeth.f. 303

Chen, W. J., Hsieh, F. C., Hsu, F. C., Tasy, Y. F., Liu, J. R., and Shih, M. C. (2014). Characterization of an insecticidal toxin and pathogenicity of Pseudomonas taiwanensis against insects. PLoS Pathog. 10:e1004288. doi: 10.1371/journal. ppat. 1004288

Chen, Z. A., Li, Z. Z., and Chen, Y. P. (2014). Jin Chanhua. Beijing: Tranditional Chinese Medicine Classics Press.

Cheng, D. Q., Ding, Z. S., Lin, M. A., Pan, P. L., and Chen, Y. T. (2006). Isolation and fermentation culture of fungi from Cordyceps sobofifera. Zhong Yao Cai 2, 99-101.

Cheng, J. W., Wang, Y. B., He, L., Qian, H., Fu, L. Z., and Li, H. B. (2012). Optimization of fermentation process for the production of intracellular polysaccharide from Paecilomyces cicadae and the immuno-stimulating activity of intracellular polysaccharide. World J. Microbiol. Biotechnol. 28, 3293-3299. doi: 10.1007/s11274-012-1140-0

Chunyu, Y. J., Lu, Z. M., Luo, Z. S., Li, S. S., Li, H., Geng, Y., et al. (2019). Promotion of metabolite synthesis in Isaria cicadae, a dominant species in the cicada flower microbiota, by cicada pupae. J. Agric. Food Chem. 67, 8476-8484. doi: 10.1021/acs.jafc.9b02705

Edgar, R. C., Haas, B. J., Clemente, J. C., Quince, C., and Knight, R. (2011). UCHIME improves sensitivity and speed of chimera detection. Bioinformatics 27, 2194-2200. doi: 10.1093/bioinformatics/btr381

Feng, L. C. (2002). Study on Shanghai Tianma hill Cordyceps Sobolifera. J. Technol. 2, $125-127$.

Feng, Y. (2008). Nitrogen allocation and partitioning in invasive and native Eupatorium species. Physiol. Plant. 132, 350-358. doi: 10.1111/j.1399-3054. 2007.01019.x

Ge, F., Xia, C. R., Li, C. R., Ding, T., Shao, Y., and Fan, M. Z. (2007). Analysis of the chemical compositions of Paecilomyces cicadae fermented mycelia and Cordyceps cicadae fruit body. Mycosystema 26, 68-75.

He, W., and Chung, H. Y. (2020). Exploring core functional microbiota related with flavor compounds involved in the fermentation of a natural fermented plain sufu (Chinese fermented soybean curd). Food Microbiol. 90:103408. doi: 10.1016/j.fm.2019.103408

He, W. W., He, L., and Li, W. Q. (2019). Research progress on chemical constituents and artificial culture of Cordyceps cicadae. Zhong Yao Cai. 42, 1691-1696.
Fan, Mingpeng Qiu, and Jiaren Zhang for help with part of our experiment on cultivating C. cicadae in the soil of a natural forest habitat.

\section{SUPPLEMENTARY MATERIAL}

The Supplementary Material for this article can be found online at: https://www.frontiersin.org/articles/10.3389/fmicb. 2021.669785/full\#supplementary-material

He, Y., Zhang, W., Peng, F., Lu, R., Zhou, H., Bao, G., et al. (2019). Metabolomic variation in wild and cultured cordyceps and mycelia of Isaria cicadae. Biomed. Chromatogr. 33:e4478. doi: 10.1002/bmc. 4478

He, Y. Q., Zhang, W. C., Peng, F., Lu, R. L., Zhou, H., Bao, G. H., et al. (2019). Metabolomic variation in wild and cultured cordyceps and mycelia of Isaria cicadae. Biomed. Chromatogr. 33:e4478. doi: 10.1002/bmc.4478

Hsu, J. H., Jhou, B. Y., Yeh, S. H., Chen, Y. L., and Chen, C. C. (2015). Healthcare functions of Cordyceps cicadae. J. Nutrit. Food Sci. 5:100432. doi: 10.4172/21559600.1000432

Hu, H. Y., Zou, X., Luo, L., Liu, A. Y., and Liang, Z. Q. (2009). Artificial culturing of Cordyceps cicadidae on living silkworm. Zhongguo Zhong Yao Za Zhi 34, 2140-2143.

Huang, W. (2008). Discussion on ecological wild cultivation of edible fungi. Edible Fungi China. 5:3334.

Kang, N., Lee, H. H., Park, I., and Seo, Y. S. (2017). Development of high cordycepin-producing Cordyceps militaris strains. Mycobiology 45, 31-38. doi: 10.5941/MYCO.2017.45.1.31

Ke, B.-J., and Lee, C.-L. (2016). Investigation on the fermentation for high adenosine and N6-(2-hydroxyethyl)-adenosine (HEA) productions of Cordyceps cicadae. New Biotechnol. 33, S206-S207. doi: 10.1016/j.nbt.2016.06. 1433

Langille, M. G. I., Zaneveld, J., Caporaso, J. G., McDonald, D., Knights, D., Reyes, J. A., et al. (2013). Predictive functional profiling of microbial communities using 16S rRNA marker gene sequences. Nat. Biotechnol. 31, 814-821. doi: 10.1038/nbt.2676

Latini, S., and Pedata, F. (2001). Adenosine in the central nervous system: release mechanisms and extracellular concentrations. J. Neurochem. 79, 463-484. doi: 10.1046/j.1471-4159.2001.00607.x

Lee, S. K., Lee, J. H., Kim, H. R., Chun, Y., Lee, J. H., Yoo, H. Y., et al. (2019). Improved cordycepin production by Cordyceps militaris KYL05 using casein hydrolysate in submerged conditions. Biomolecules 9:461. doi: 10.3390/ biom 9090461

Li, L., Abu Al-Soud, W., Bergmark, L., Riber, L., Hansen, L. H., Magid, J., et al. (2013). Investigating the diversity of Pseudomonas spp. in soil using culture dependent and independent techniques. Curr. Microbiol. 67, 423-430. doi: 10. 1007/s00284-013-0382-x

Li, L., Zhang, T., Li, C., Xie, L., Li, N., Hou, T., et al. (2019). Potential therapeutic effects of Cordyceps cicadae and Paecilomyces cicadae on adenine-induced chronic renal failure in rats and their phytochemical analysis. Drug Des. Devel. Ther. 13, 103-117. doi: 10.2147/dddt.S180543

Lin, S., Liu, Z. Q., Xue, Y. P., Baker, P. J., Wu, H., Xu, F., et al. (2016). Biosynthetic pathway analysis for improving the cordycepin and cordycepic acid production in Hirsutella sinensis. Appl. Biochem. Biotechnol. 179, 633-649. doi: 10.1007/ s12010-016-2020-0

Liu, A. Y. (2012). Research and Application of Cordyceps cicadae Resources in China. Guiyang: Guizhou Science and Technology Publishing House.

Liu, S. Q., Wen, L., Xia, M., and Jiang, N. (2008). Determination of the active ingredient produced in the artificial cultivated Cordyceps sobolifera. J. Anhu. Agric. Sci. 36, 429-467.

Liu, T., Zhang, H., Shi, X., Ma, W., Zhang, Y., Liu, Z., et al. (2018). Illumina MiSeq sequencing investigation of Chanhua (Cordyceps cicadae Shing) fungal community structures in different regions. J. Trad. Chin. Med. Sci. 5, 206-212. doi: $10.1016 /$ j.jtcms.2017.07.008 
Lu, J., Gu, G., Hao, L., Jin, Z., and Wang, X. (2016). Characterization and in vitro antioxidant activity of a polysaccharide from Cordyceps sobolifera. J. Food Process. Preserv. 40, 447-452. doi: 10.1111/jfpp.12622

Magoc, T., and Salzberg, S. L. (2011). FLASH: fast length adjustment of short reads to improve genome assemblies. Bioinformatics 27, 2957-2963. doi: 10.1093/ bioinformatics/btr507

Mao, X. B., Eksriwong, T., Chauvatcharin, S., and Zhong, J. J. (2005). Optimization of carbon source and carbon/nitrogen ratio for cordycepin production by submerged cultivation of medicinal mushroom Cordyceps militaris. Process Biochem. 40, 1667-1672. doi: 10.1016/j.procbio.2004.06.046

Mau, J. L., Lin, H. C., and Chen, C. C. (2001). Non-volatile components of several medicinal mushrooms. Food Res. Int. 34, 521-526. doi: 10.1016/s0963-9969(01) 00067-9

Mou, D., Zeng, Z. Y., Zhong, W. L., Zhou, J. X., Qu, J. J., and Zou, X. (2021). Bacterial community structure and function of Cordyceps cicadae microecosystem in Guiyang. Act. Microbiol. Sin. doi: 10.13343/j.cnki.wsxb. 20200540 [Epub ahead of print],

Mueller, E. A., Wisnoski, N. I., Peralta, A. L., and Lennon, J. T. (2020). Microbial rescue effects: how microbiomes can save hosts from extinction. Funct. Ecol. 34, 2055-2064. doi: 10.1111/1365-2435.13493

Nakav, S., Chaimovitz, C., Sufaro, Y., Lewis, E. C., Shaked, G., Czeiger, D., et al. (2008). Anti-inflammatory preconditioning by agonists of adenosine A1 receptor. PLoS One 3:e2107. doi: 10.1371/journal.pone.0002107

Nxumalo, W., Elateeq, A. A., and Sun, Y. (2020). Can Cordyceps cicadae be used as an alternative to Cordyceps militaris and Cordyceps sinensis? - a review. J. Ethnopharmacol. 257:112879. doi: 10.1016/j.jep.2020.112879

Olatunji, O. J., Feng, Y., Olatunji, O. O., Tang, J., Ouyang, Z., and Su, Z. (2016a). Cordycepin protects PC12 cells against 6-hydroxydopamine induced neurotoxicity via its antioxidant properties. Biomed. Pharmacother. 81, 7-14. doi: 10.1016/j.biopha.2016.03.009

Olatunji, O. J., Feng, Y., Olatunji, O. O., Tang, J., Ouyang, Z., Su, Z., et al. (2016b). Neuroprotective effects of adenosine isolated from Cordyceps cicadae against oxidative and ER stress damages induced by glutamate in PC12 cells. Environ. Toxicol. Pharmacol. 44, 53-61. doi: 10.1016/j.etap.2016.02.009

Qu, Q. S., Yang, F., Zhao, C. Y., and Shi, X. Y. (2019a). Analysis of the bacteria community in wild Cordyceps cicadae and its influence on the production of HEA and nucleosides in Cordyceps cicadae. J. Appl. Microbiol. 127, 1759-1767. doi: 10.1111/jam.14432

Qu, Q. S., Yang, F., Zhao, C. Y., Wang, J. R., Zeng, J. H., and Shi, X. Y. (2019b). Isolation and diversity of Cordyceps cicadae endophytic bacteria in Anhui and Zhejiang. Zhong Yao Cai. 42, 992-999. doi: 10.13863/j.issn1001-4454.2019.05. 009

Quast, C., Pruesse, E., Yilmaz, P., Gerken, J., Schweer, T., Yarza, P., et al. (2013). The SILVA ribosomal RNA gene database project: improved data processing and web-based tools. Nucleic Acids Res. 41, D590-D596. doi: 10.1093/nar/gks1219

San, W. J., Li, T. H., and Jiang, Z. D. (2010). Determination of cordyceps acid in Guangdong by colorimetry. Edible Fungi. 32, 73-74.

Simon, J., Gleadow, R. M., and Woodrow, I. E. (2010). Allocation of nitrogen to chemical defence and plant functional traits is constrained by soil N. Tree. Physiol. 30, 1111-1117. doi: 10.1093/treephys/tpq049

Sun, Y. F., Kmonickova, E., Han, R. L., Zhou, W., Yang, K. B., Lu, H. F., et al. (2019). Comprehensive evaluation of wild Cordyceps cicadae from different geographical origins by TOPSIS method based on the macroscopic infrared spectroscopy (IR) fingerprint. Spectrochim. Acta A Mol. Biomol. Spectrosc. 214, 252-260. doi: 10.1016/j.saa.2019.02.031

Wang, Y., He, P., He, L., Huang, Q., Cheng, J., Li, W., et al. (2019). Structural elucidation, antioxidant and immunomodulatory activities of a novel hetero polysaccharide from cultured Paecilomyces cicadae (Miquel.) Samson. Carbohydr. Polym. 216, 270-281. doi: 10.1016/j.carbpol.2019.03.104

Wu, H., Rao, Z.-C., Cao, L., De Clercq, P., and Han, R.-C. (2020). Infection of Ophiocordyceps sinensis fungus causes dramatic changes in the microbiota of its thitarodes host. Front. Microbiol. 11:577268. doi: 10.3389/fmicb.2020.57 7268

Xia, F., Liu, Y., Shen, G. R., Guo, L. X., and Zhou, X. W. (2015). Investigation and analysis of microbiological communities in natural Ophiocordyceps sinensis. Can. J. Microbiol. 61, 104-111. doi: 10.1139/cjm-2014-0610

Yu, M., Li, Y., Banakar, S. P., Liu, L., Shao, C., Li, Z., et al. (2019). New metabolites from the co-culture of marine-derived Actinomycete Streptomyces rochei MB037 and fungus Rhinocladiella similis 35. Front. Microbiol. 10:915. doi: 10.3389/fmicb.2019.00915

Zang, J., Xu, Y., Xia, W., Regenstein, J. M., Yu, D., Yang, F., et al. (2020). Correlations between microbiota succession and flavor formation during fermentation of Chinese low-salt fermented common carp (Cyprinus carpio L.) inoculated with mixed starter cultures. Food Microbiol. 90:103487. doi: 10.1016/ j.fm.2020.103487

Zeng, W. B., Chang, C. X., Li, J. P., Wang, Y. B., Dai, Y. D., and Yu, H. (2017). Micro-morphological variation pattern of Isaria cicadae. Acta Microbiol. Sin. 57, 350-362.

Zeng, W. B., Wang, Y. B., Yu, H., Dai, Y. D., and Chen, Z. H. (2015). Population distribution of Isaria cicadae and its habitat investigation. Edible Fungi China 34, 50-56.

Zeng, W. B., Yu, H., Ge, F., Yang, J. Y., Chen, Z. H., Wang, Y. B., et al. (2014). Distribution of nucleosides in populations of Cordyceps cicadae. Molecules 19, 6123-6141.

Zeng, Z. Y., Xu, Z. S., Mou, D., Xu, S. H., Du, F., and Zhou, J. X. (2019). Analyses on the structure and ecological function of fungal community in sclerotia, external mycelial layer covering ossified cicada epidermis and habitat soil of Isaria cicadae. Mycosystema 38, 1710-1723.

Zhang, H. M., Shi, X. S., Liu, T. F., Ma, W., Zhang, Y. C., and Shi, X. Y. (2017). Comparing research on contents of adenosine, cordycepin and ergosterol in Cordyceps cicadae. Glob. Trad. Chin. Med. 10, 297-301.

Zhang, H. X., Gao, X. H., Chen, W., and Huang, K. H. (2012). Levels of selected chemical components in Cordyceps sobolifera fruit bodies cultivated artificially and collected from the wild. Acta Edulis Fungi 19, 59-62.

Zhang, Q., Olatunji, O. J., Chen, H., Tola, A. J., and Oluwaniyi, O. O. (2018). Evaluation of the anti-diabetic activity of polysaccharide from Cordyceps cicadae in experimental diabetic rats. Chem. Biodivers. 15:e1800219. doi: 10. 1002/cbdv.201800219

Zhang, X., Hu, Q., and Weng, Q. (2019). Secondary metabolites (SMs) of Isaria cicadae and Isaria tenuipes. RSC Adv. 9, 172-184. doi: 10.1039/C8RA0 9039D

Zhang, Y., Xiao, X. J., Zhang, M. Y., Xiong, J., and Huang, Z. X. (2016). Key technique for artificial rearing of Cordyceps militaris. Guizhou Agric. Sci. 44, 65-68.

Zhang, Y., Zhang, S., Wang, M., Bai, F., and Liu, X. (2010). High diversity of the fungal community structure in naturally-occurring Ophiocordyceps sinensis. PLoS One 5:e15570. doi: 10.1371/journal.pone.00 15570

Zhou, J. X., Yu, L. F., Zhang, J., Zhang, X. M., Xue, Y., Liu, J., et al. (2020). Characterization of the core microbiome in tobacco leaves during aging. MicrobiologyOpen 9:e984. doi: 10.1002/mbo3.984

Conflict of Interest: The authors declare that the research was conducted in the absence of any commercial or financial relationships that could be construed as a potential conflict of interest.

Copyright (c) 2021 Zeng, Mou, Luo, Zhong, Duan and Zou. This is an open-access article distributed under the terms of the Creative Commons Attribution License (CC BY). The use, distribution or reproduction in other forums is permitted, provided the original author(s) and the copyright owner(s) are credited and that the original publication in this journal is cited, in accordance with accepted academic practice. No use, distribution or reproduction is permitted which does not comply with these terms. 